An embedded boundary method for the wave equation with discontinuous coefficients

H. O. Kreiss, N. A. Petersson

September 28, 2005

SIAM Journal of Scientific Computing 
This document was prepared as an account of work sponsored by an agency of the United States Government. Neither the United States Government nor the University of California nor any of their employees, makes any warranty, express or implied, or assumes any legal liability or responsibility for the accuracy, completeness, or usefulness of any information, apparatus, product, or process disclosed, or represents that its use would not infringe privately owned rights. Reference herein to any specific commercial product, process, or service by trade name, trademark, manufacturer, or otherwise, does not necessarily constitute or imply its endorsement, recommendation, or favoring by the United States Government or the University of California. The views and opinions of authors expressed herein do not necessarily state or reflect those of the United States Government or the University of California, and shall not be used for advertising or product endorsement purposes. 


\title{
An embedded boundary method for the wave equation with discontinuous coefficients*
}

\author{
Heinz-Otto Kreiss ${ }^{\dagger} \quad$ N. Anders Petersson ${ }^{\ddagger}$
}

September 26, 2005

\begin{abstract}
A second order accurate embedded boundary method for the two-dimensional wave equation with discontinuous wave propagation speed is described. The wave equation is discretized on a Cartesian grid with constant grid size and the interface (across which the wave speed is discontinuous) is allowed to intersect the mesh in an arbitrary fashion. By using ghost points on either side of the interface, previous embedded boundary techniques for the Neumann and Dirichlet problems are generalized to satisfy the jump conditions across the interface to second order accuracy. The resulting discretization of the jump conditions has the desirable property that each ghost point can be updated independently of all other ghost points, resulting in a fully explicit time-integration method. Numerical examples are given where the method is used to study electro-magnetic scattering of a plane wave by a dielectric cylinder. The numerical solutions are evaluated against the analytical solution due to Mie, and point-wise second order accuracy is confirmed.
\end{abstract}

\section{Introduction}

This paper describes a second order accurate Cartesian embedded boundary method for the two-dimensional wave equation with discontinuous wave propagation speed. Motivated by wave propagation problems from applications like seismology, acoustics, and general relativity, where the underlying differential equations are systems of second-order hyperbolic partial differential equations, we develop a numerical method that directly discretizes the wave equation in second order formulation. This approach extends previous research $[1,2,3]$ to the case of discontinuous coefficients.

${ }^{*}$ This work was performed under the auspices of the U.S. Department of Energy by the University of California, Lawrence Livermore National Laboratory, under contract W-7405-Eng-48.

†Träskö-Storö Institute of Mathematics, Stockholm, Sweden (hokreiss@nada.kth.se).

$\ddagger$ Center for Applied Scientific Computing, LLNL, Livermore, California (andersp@llnl.gov). 
For every second-order hyperbolic system, there is an equivalent but larger first-order system. For example, two-dimensional acoustic wave propagation is governed by a scalar second-order wave equation for the pressure, or by a system of three first-order hyperbolic equations governing the two velocity components and the pressure. Most previous numerical methods for this type of problems have focused on the first-order formulation $[4,5,6]$. For linear wave propagation, a staggered grid is often used to avoid complications with stability of extra numerical boundary conditions [7] and spurious waves traveling in the wrong direction [8]. However, Cartesian staggered grid discretizations are difficult to generalize to handle complex geometries, that is, boundaries that intersect the grid in an arbitrary way.

The accuracy of a finite difference approximation of the wave equation with discontinuous coefficients was analyzed by Brown [9]. For the one-dimensional case, he proved that the amplitude error in reflected and transmitted waves is determined by the accuracy by which the jump conditions are discretized, while the phase error is determined by the accuracy of the discretization in the interior of the domain. In the one-dimensional case, Tikhonov and Samarskiı̌'s [10] averaging formula was used to obtain a second order accurate approximation without explicitly discretizing the jump conditions. Numerical calculations indicated a significant benefit of combining the second order treatment of the jump conditions with a fourth order method in the interior of the domain. Unfortunately, it is not known how to generalize the averaging formula to the two-dimensional case when the discontinuity is not aligned with the grid, and the combinations of a first order treatment of the jump conditions with a second or fourth order formula away from the interface gave less impressive results than in the one-dimensional case.

A fully second order immersed interface method for solving the two-dimensional acoustic wave equation with discontinuous coefficients was developed by Zhang and LeVeque [11]. Here, the problem was written as a hyperbolic system of three first order equations and special difference formulas were developed near the interface, which take the location of the interface and the jump conditions into account to achieve second order accuracy. For each grid point next to the interface, a linear system with 54 equations for 54 unknowns had to be solved to find the values of the coefficients in the local difference formula. For more complicated hyperbolic systems (such as the elastic wave equation), even larger systems of equations must be solved to set up the coefficients.

In this paper, we consider the scalar second-order wave equation in a two-dimensional domain $\Omega=\Omega_{I} \cup \Omega_{I I}$, with a piecewise constant coefficient $\rho(\boldsymbol{x})>0$,

$$
\rho(\boldsymbol{x})= \begin{cases}\rho_{I}, & \boldsymbol{x} \in \Omega_{I}, \\ \rho_{I I}, & \boldsymbol{x} \in \Omega_{I I} .\end{cases}
$$


Let $u(\boldsymbol{x}, t)$ and $w(\boldsymbol{x}, t)$ denote the solutions in the sub-domains $\Omega_{I}$ and $\Omega_{I I}$, satisfying

$$
\begin{array}{rlrl}
u_{t t} & =\frac{1}{\rho_{I}} \Delta u+F(\boldsymbol{x}, t), & & \boldsymbol{x} \in \Omega_{I}, \\
w_{t t} & =\frac{1}{\rho_{I I}} \Delta w+F(\boldsymbol{x}, t), & & t \geq 0, \\
& \boldsymbol{x} \in \Omega_{I I}, & t \geq 0 . \\
u(\boldsymbol{x}, 0)=U_{0}(\boldsymbol{x}), & u_{t}(\boldsymbol{x}, 0) & =U_{1}(\boldsymbol{x}), & \boldsymbol{x} \in \Omega_{I}, \\
w(\boldsymbol{x}, 0)=W_{0}(\boldsymbol{x}), & w_{t}(\boldsymbol{x}, 0) & =W_{1}(\boldsymbol{x}), & \boldsymbol{x} \in \Omega_{I I} .
\end{array}
$$

Here, $F(\boldsymbol{x}, t)$ is a forcing function. Let $\Gamma$ be the smooth interface between $\Omega_{I}$ and $\Omega_{I I}$, across which the solutions are coupled by the jump conditions

$$
\begin{aligned}
& u=w, \quad \boldsymbol{x} \in \Gamma, \quad t \geq 0, \\
& \frac{1}{\rho_{I}} \frac{\partial u}{\partial n}=-\frac{1}{\rho_{I I}} \frac{\partial w}{\partial n}, \quad \boldsymbol{x} \in \Gamma, \quad t \geq 0 .
\end{aligned}
$$

Note that the normal derivatives are taken outwards from both $\Omega_{I}$ and $\Omega_{I I}$. Hence the minus sign in the latter equation.

For the purpose of our discussion, we assume that $\Omega_{I}$ is a bounded domain inside $\Omega_{I I}$, and that $\Omega_{I I}$ has a rectangular outer boundary where Dirichlet or Neumann boundary conditions are enforced. The interface between $\Omega_{I}$ and $\Omega_{I I}$ can however have an arbitrary smooth shape. As will be demonstrated below, the method can be generalized to several subdomains. Non-rectangular outer boundaries can be handled using the embedded boundary techniques in [2] and [3].

We discretize the two-dimensional wave equations on a Cartesian grid $\boldsymbol{x}_{i, j}=(i h, j h)$ in space, where $h>0$ is the grid size, and let $t_{n}=n \delta_{t}, n=0,1,2, \ldots$ denote the time-discretization with step size $\delta_{t}>0$. We take $u_{i, j}^{n}$ and $w_{i, j}^{n}$ to be the difference approximations of $u\left(x_{i}, y_{j}, t_{n}\right)$ and $w\left(x_{i}, y_{j}, t_{n}\right)$, respectively. At all grid points interior to $\Omega_{I}$, a second order accurate approximation of the Laplacian of $u$ is given by

$$
\Delta_{h} u_{i, j}^{n}=: \frac{1}{h^{2}}\left(u_{i+1, j}^{n}+u_{i-1, j}^{n}+u_{i, j+1}^{n}+u_{i, j-1}^{n}-4 u_{i, j}^{n}\right), \quad \boldsymbol{x}_{i, j} \in \Omega_{I}
$$

In order to form $(7)$ at all grid points in $\Omega_{I}$, we also define $u_{i, j}^{n}$ at the set of ghost points $G_{I}$ :

$$
G_{I}=\left\{(i, j), \boldsymbol{x}_{i, j} \notin \Omega_{I}, \text { but at least one of } \boldsymbol{x}_{i \pm 1, j} \in \Omega_{I} \text { or } \boldsymbol{x}_{i, j \pm 1} \in \Omega_{I}\right\} .
$$

A corresponding formula is used to approximate the Laplacian of $w$ at all interior grid points of $\Omega_{I I}$ using a corresponding set of ghost points $G_{I I}$.

The solution at the ghost points are determined by the jump conditions (5) and (6). We start by considering the ghost point $u_{i, j}$, see Figure 1 . We can derive second order approximations for the value and normal derivative on the boundary using Lagrange 


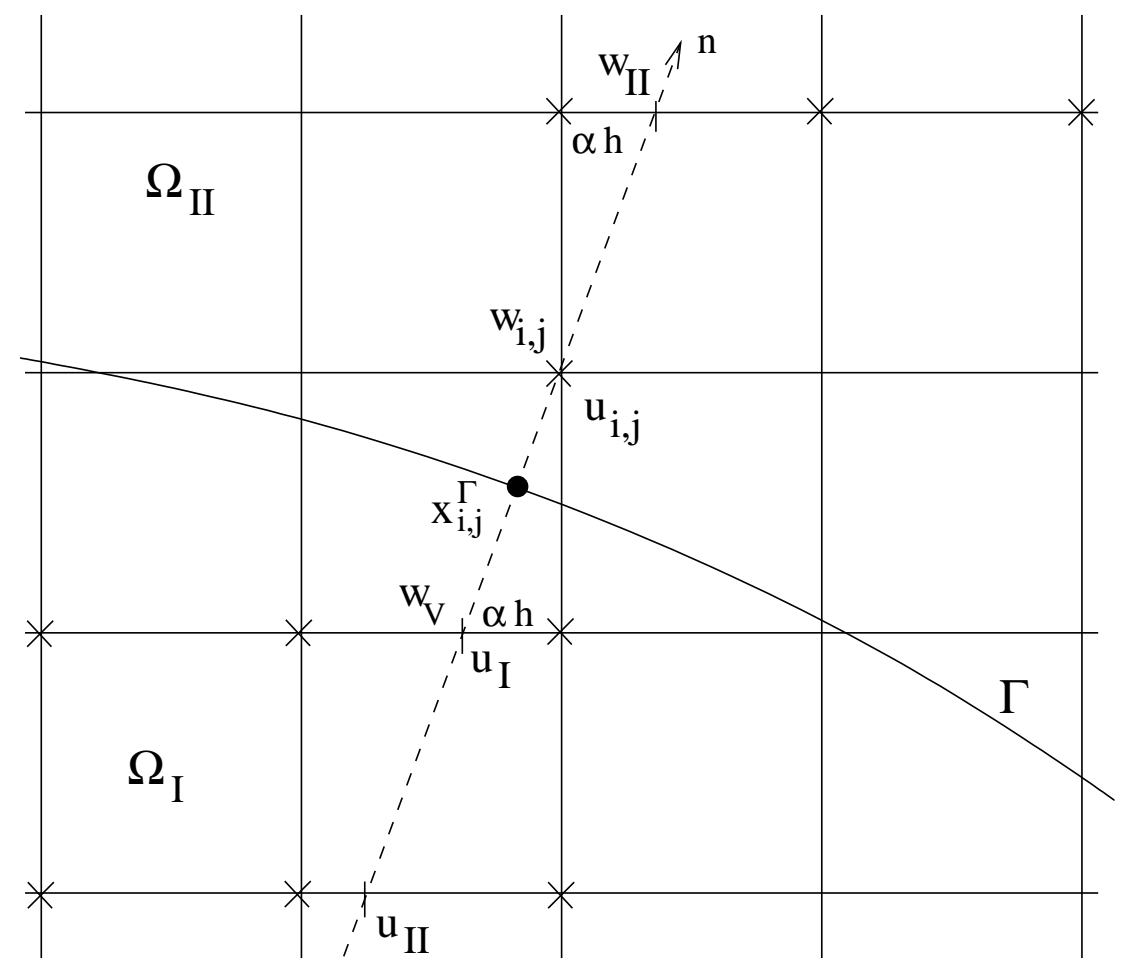

Figure 1: The points used for discretizing the jump conditions.

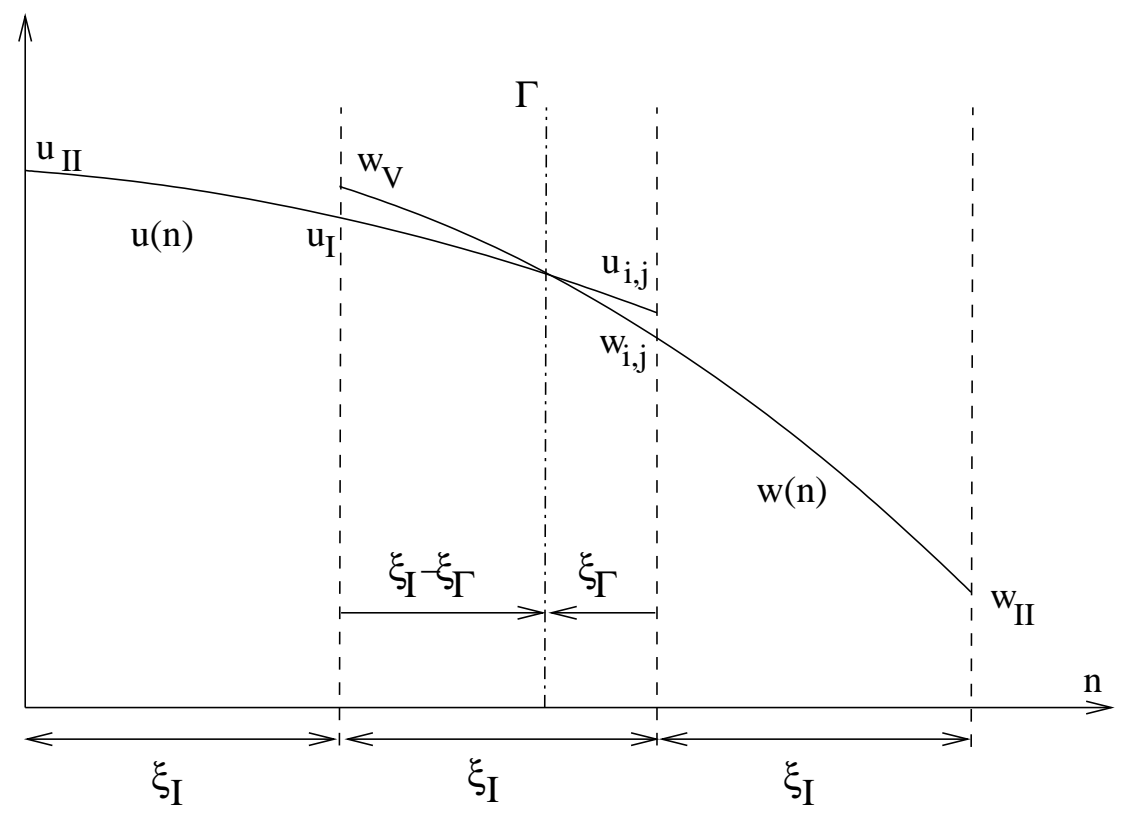

Figure 2: The solution along the normal to the interface is continuous, but its first derivative is discontinuous. 
interpolation between $u_{i, j}, u_{I}$, and $u_{I I}$ (see Figure 2),

$$
\begin{array}{ll}
B_{i, j}^{D} u=: g_{0}^{D}\left(\xi_{\Gamma}\right) u_{i, j}+g_{I}^{D}\left(\xi_{\Gamma}\right) u_{I}+g_{I I}^{D}\left(\xi_{\Gamma}\right) u_{I I}=u\left(\boldsymbol{x}_{i, j}^{\Gamma}\right)+\mathcal{O}\left(h^{2}\right), & (i, j) \in G_{I}, \\
B_{i, j}^{N} u=: g_{0}^{N}\left(\xi_{\Gamma}\right) u_{i, j}+g_{I}^{N}\left(\xi_{\Gamma}\right) u_{I}+g_{I I}^{N}\left(\xi_{\Gamma}\right) u_{I I}=\frac{\partial u}{\partial n}\left(\boldsymbol{x}_{i, j}^{\Gamma}\right)+\mathcal{O}\left(h^{2}\right), \quad(i, j) \in G_{I} .
\end{array}
$$

The values $u_{I}$ and $u_{I I}$ are obtained by Lagrange interpolation, in this case, along the horizontal grid lines $y_{j-1}$ and $y_{j-2}$,

$$
\begin{gathered}
u_{I}^{n}=r_{0} u_{i, j-1}^{n}+r_{1} u_{i-1, j-1}^{n}+r_{2} u_{i-2, j-1}^{n}, \\
u_{I I}^{n}=r_{3} u_{i, j-2}^{n}+r_{4} u_{i-1, j-2}^{n}+r_{5} u_{i-2, j-2}^{n} .
\end{gathered}
$$

The coefficients $g^{D}$ and $g^{N}$ are given in Section 4 .

To derive corresponding formulas for $w$ along the same normal, we introduce the virtual ghost point value $w_{v}$, defined at the same location as $u_{I}$, see Figure 1 . Since $w_{v}$ is outside $\Omega_{I I}$ and it is in general located in between grid points, this value is only used to form the discrete jump conditions. Lagrange interpolation between $w_{v}, w_{i, j}$, and $w_{I I}$ (see Figure 2) gives for $(i, j) \in G_{I}$,

$$
\begin{aligned}
& V_{i, j}^{D} w=: g_{0}^{D}\left(\xi_{I}-\xi_{\Gamma}\right) w_{v}+g_{I}^{D}\left(\xi_{I}-\xi_{\Gamma}\right) w_{i, j}+g_{I I}^{D}\left(\xi_{I}-\xi_{\Gamma}\right) w_{I I}=w\left(\boldsymbol{x}_{i, j}^{\Gamma}\right)+\mathcal{O}\left(h^{2}\right), \\
& V_{i, j}^{N} w=: g_{0}^{N}\left(\xi_{I}-\xi_{\Gamma}\right) w_{v}+g_{I}^{N}\left(\xi_{I}-\xi_{\Gamma}\right) w_{i, j}+g_{I I}^{N}\left(\xi_{I}-\xi_{\Gamma}\right) w_{I I}=\frac{\partial w}{\partial n}\left(\boldsymbol{x}_{i, j}^{\Gamma}\right)+\mathcal{O}\left(h^{2}\right) .
\end{aligned}
$$

Note that there is a symmetry between the interpolation formulas for $u_{I}$ and $w_{I I}$ that simplifies the calculation of $w_{I I}$. If the normal going through $\boldsymbol{x}_{i, j}$ intersects the horizontal grid line $y=y_{j-1}$ at $x=x_{i}-\alpha h$, the extension of the same normal will intersect the grid line $y=y_{j+1}$ at $x=x_{i}+\alpha h$. The Lagrange interpolation formula for $w_{I I}^{n}$ therefore becomes

$$
w_{I I}^{n}=r_{0} w_{i, j+1}^{n}+r_{1} w_{i+1, j+1}^{n}+r_{2} w_{i+2, j+1}^{n} .
$$

Inserting the discrete approximations of the value and normal derivatives of $u$ and $w$ into the jump conditions (5), (6), results in the discretized jump conditions:

$$
\begin{aligned}
B_{i, j}^{D} u^{n} & =V_{i, j}^{D} w^{n}, \\
\frac{1}{\rho_{I}} B_{i, j}^{N} u^{n} & =-\frac{1}{\rho_{I I}} V_{i, j}^{N} w^{n} .
\end{aligned}
$$

Hence, we get a $2 \times 2$ linear system for the unknowns $\left(u_{i, j}^{n}, w_{v}^{n}\right)$ :

$$
\begin{aligned}
& \left(\begin{array}{cc}
g_{0}^{D}\left(\xi_{\Gamma}\right) & -g_{0}^{D}\left(\xi_{I}-\xi_{\Gamma}\right) \\
g_{0}^{N}\left(\xi_{\Gamma}\right) / \rho_{I} & g_{0}^{N}\left(\xi_{I}-\xi_{\Gamma}\right) / \rho_{I I}
\end{array}\right)\left(\begin{array}{c}
u_{i, j}^{n} \\
w_{v}^{n}
\end{array}\right)= \\
& \quad\left(\begin{array}{c}
g_{I}^{D}\left(\xi_{I}-\xi_{\Gamma}\right) w_{i, j}^{n}+g_{I I}^{D}\left(\xi_{I}-\xi_{\Gamma}\right) w_{I I}^{n}-g_{I}^{D}\left(\xi_{\Gamma}\right) u_{I}^{n}-g_{I I}^{D}\left(\xi_{\Gamma}\right) u_{I I}^{n} \\
-\left(g_{I}^{N}\left(\xi_{I}-\xi_{\Gamma}\right) w_{i, j}^{n}+g_{I I}^{N}\left(\xi_{I}-\xi_{\Gamma}\right) w_{I I}^{n}\right) / \rho_{I I}-\left(g_{I}^{N}\left(\xi_{\Gamma}\right) u_{I}^{n}+g_{I I}^{N}\left(\xi_{\Gamma}\right) u_{I I}^{n}\right) / \rho_{I}
\end{array}\right) .
\end{aligned}
$$


We solve this system for $u_{i, j}^{n}$ and ignore the virtual ghost point value $w_{v}^{n}$. A formula for the $w$-ghost points can be derived in a similar way.

Note that this discretization of the jump conditions has the desirable property that each ghost point can be updated independently of all other ghost points. Hence, there is no coupling along the boundary.

The remainder of the paper is organized as follows. In Section 2, we prove that the discretization of the jump conditions is stable in the one-dimensional case. A simplified stability argument, based on a modified equation model, is presented in Section 3. The solvability of the discrete jump conditions in the two-dimensional setting is demonstrated in Section 4. Section 5 discusses time-integration and a generalization of the $A^{T} A$ dissipation operator (see [2]), which is used to stabilize the scheme for long time integrations. In Section 6, we use the method to study electro-magnetic scattering of a plane wave by a dielectric cylinder, where there is a century old analytical solution due to Mie [12]. The method is finally applied to a more complicated scattering problem consisting of many subdomains.

\section{Stability}

We start by considering the one-dimensional wave equation with discontinuous wave propagation speed,

$$
\begin{aligned}
& u_{t t}=u_{x x}, \quad x_{\min } \leq x \leq 0, \quad t \geq 0, \\
& w_{t t}=c^{2} w_{x x}, \quad 0 \leq x \leq x_{\max }, \quad t \geq 0, \\
& u(x, 0)=U_{0}(x), \quad u_{t}(x, 0)=U_{1}(x), \quad x_{\min } \leq x \leq 0, \\
& w(x, 0)=W_{0}(x), \quad w_{t}(x, 0)=W_{1}(x), \quad 0 \leq x \leq x_{\max },
\end{aligned}
$$

where $c^{2}>0, x_{\min }<0, x_{\max }>0$, subject to the Dirichlet boundary conditions

$$
u\left(x_{\min }, t\right)=0, \quad w\left(x_{\max }, t\right)=0, \quad t \geq 0
$$

At the interface, the jump conditions are

$$
\begin{aligned}
u(0, t) & =w(0, t), \\
u_{x}(0, t) & =c^{2} w_{x}(0, t) .
\end{aligned}
$$

Using the notation in Figure 3, we discretize the problem in space on a uniform grid $x_{\nu}=-\alpha h+\nu h$, with grid size $h>0$, and denote a grid function by $u_{\nu}(t)=u\left(x_{\nu}, t\right)$. Divided difference operators are defined by $D_{+}^{x} u_{\nu}=\left(u_{\nu+1}-u_{\nu}\right) / h$ and $D_{-}^{x} u_{\nu}=D_{+}^{x} u_{\nu-1}$. To model the two-dimensional case, we shift the grid so the discontinuity in the wave speed does not coincide with a grid point, i.e., $0<\alpha<1$. We want to focus the discussion on the stability of the discretization of the jump conditions, and we take $x_{\min }$ and $x_{\max }$ to 


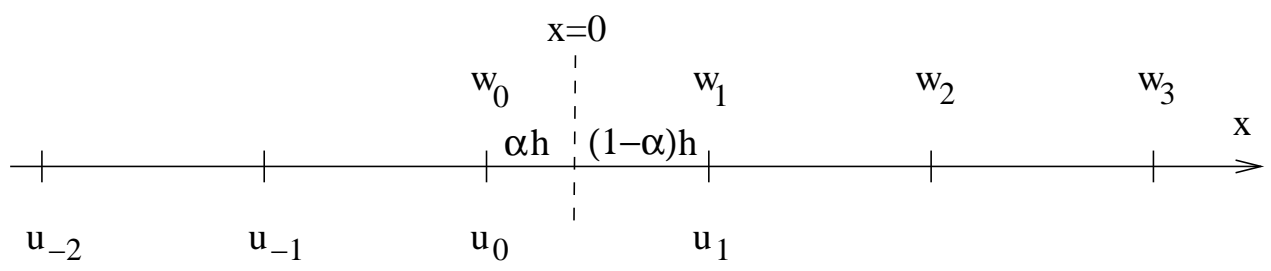

Figure 3: Notation for the one-dimensional problem.

coincide with grid points, i.e., $x_{\min }=x_{-N}$ and $x_{\max }=x_{N}$. The semi-discrete problem becomes

$$
\begin{gathered}
\frac{d^{2} u_{\nu}}{d t^{2}}=D_{+}^{x} D_{-}^{x} u_{\nu}, \quad \nu=0,-1,-2, \ldots,-N+1, \\
\frac{d^{2} w_{\nu}}{d t^{2}}=c^{2} D_{+}^{x} D_{-}^{x} w_{\nu}, \quad \quad \nu=1,2,3, \ldots, N-1,
\end{gathered}
$$

subject to Dirichlet boundary conditions

$$
u_{-N}=0, \quad w_{N}=0, \quad t \geq 0 .
$$

We discretize the jump conditions to second order accuracy by

$$
\begin{aligned}
(1-\alpha) u_{0}+\alpha u_{1} & =(1-\alpha) w_{0}+\alpha w_{1} \\
D_{+}^{x} u_{0}+h\left(\alpha-\frac{1}{2}\right) D_{+}^{x} D_{-}^{x} u_{0} & =c^{2}\left(D_{+}^{x} w_{0}+h\left(\alpha-\frac{1}{2}\right) D_{+}^{x} D_{-}^{x} w_{1}\right) .
\end{aligned}
$$

By using (22) and (23), we can write (25), (26) as

$$
\begin{aligned}
\alpha u_{1}+(\alpha-1) w_{0} & =(\alpha-1) u_{0}+\alpha w_{1} \\
u_{1}+c^{2} w_{0} & =u_{0}+c^{2} w_{1}+h^{2}\left(\alpha-\frac{1}{2}\right)\left(\frac{d^{2} w_{1}}{d t^{2}}-\frac{d^{2} u_{0}}{d t^{2}}\right) .
\end{aligned}
$$

Solving for $w_{0}$ and $u_{1}$ yields

$$
\begin{aligned}
& w_{0}=\frac{1}{1-\alpha\left(1-c^{2}\right)}\left(u_{0}-\alpha\left(1-c^{2}\right) w_{1}+h^{2} \alpha\left(\alpha-\frac{1}{2}\right)\left(\frac{d^{2} w_{1}}{d t^{2}}-\frac{d^{2} u_{0}}{d t^{2}}\right)\right), \\
& u_{1}=\frac{1}{1-\alpha\left(1-c^{2}\right)}\left(c^{2} w_{1}+(1-\alpha)\left(1-c^{2}\right) u_{0}+\right. \\
& \left.h^{2}(1-\alpha)\left(\alpha-\frac{1}{2}\right)\left(\frac{d^{2} w_{1}}{d t^{2}}-\frac{d^{2} u_{0}}{d t^{2}}\right)\right) .
\end{aligned}
$$

By using (27)-(28) we can eliminate $w_{0}, u_{1}$ from (22)-(23), and obtain

$$
\begin{aligned}
& b_{11} \frac{d^{2} u_{0}}{d t^{2}}+b_{12} \frac{d^{2} w_{1}}{d t^{2}}=\frac{1}{h^{2}}\left(a_{10} u_{-1}+a_{11} u_{0}+a_{12} w_{1}\right) \\
& b_{21} \frac{d^{2} u_{0}}{d t^{2}}+b_{22} \frac{d^{2} w_{1}}{d t^{2}}=\frac{1}{h^{2}}\left(a_{21} u_{0}+a_{22} w_{1}+a_{23} w_{2}\right) .
\end{aligned}
$$


Here,

$$
\begin{aligned}
& b_{11}=1-\alpha\left(1-c^{2}\right)+\left(\alpha-\frac{1}{2}\right)(1-\alpha), \quad b_{12}=-\left(\alpha-\frac{1}{2}\right)(1-\alpha), \\
& b_{21}=\alpha\left(\alpha-\frac{1}{2}\right), \quad b_{22}=\frac{1}{c^{2}}\left(1-\alpha\left(1-c^{2}\right)-c^{2} \alpha\left(\alpha-\frac{1}{2}\right)\right) .
\end{aligned}
$$

and

$$
\begin{array}{lll}
a_{10}=1-\alpha\left(1-c^{2}\right), & a_{11}=-2+(1+\alpha)\left(1-c^{2}\right), & a_{12}=c^{2}, \\
a_{21}=1, & a_{22}=-2+\alpha\left(1-c^{2}\right), & a_{23}=1-\alpha\left(1-c^{2}\right) .
\end{array}
$$

We can write the semi-discrete problem in matrix form,

$$
\begin{aligned}
& \left(\begin{array}{cccccccc}
1 & 0 & & & & & & \\
& \ddots & & & & & & \\
& 0 & 1 & 0 & & & & \\
& & 0 & b_{11} & b_{12} & & & \\
& & & b_{21} & b_{22} & 0 & & \\
& & & 0 & 1 / c^{2} & 0 & \\
& & & & & \ddots & \\
& & & & & 0 & 1 / c^{2}
\end{array}\right)\left(\begin{array}{c}
d^{2} \\
d t^{2} \\
u_{-1} \\
u_{0} \\
w_{1} \\
w_{2} \\
\vdots \\
w_{N-1}
\end{array}\right)= \\
& \frac{1}{h^{2}}\left(\begin{array}{ccccccc}
-2 & 1 & & & & & \\
& \ddots & & & & & \\
& 1 & -2 & 1 & & & \\
& & a_{10} & a_{11} & a_{12} & & \\
& & & a_{21} & a_{22} & a_{23} & \\
& & & & 1 & -2 & 1 \\
& & & & & \ddots & \\
& & & & 1 & -2
\end{array}\right)\left(\begin{array}{c}
u_{-N+1} \\
\vdots \\
u_{-1} \\
u_{0} \\
w_{1} \\
w_{2} \\
\vdots \\
w_{N-1}
\end{array}\right)
\end{aligned}
$$

which we write as

$$
\mathcal{B} \mathbf{w}_{t t}=\frac{1}{h^{2}} \mathcal{A} \mathbf{w}
$$


By (31), (32), the matrix $\mathcal{A}$ is negative diagonally dominant and the off-diagonal elements are positive. Therefore it can be symmetrized by a diagonal scaling $\mathcal{D}>0$,

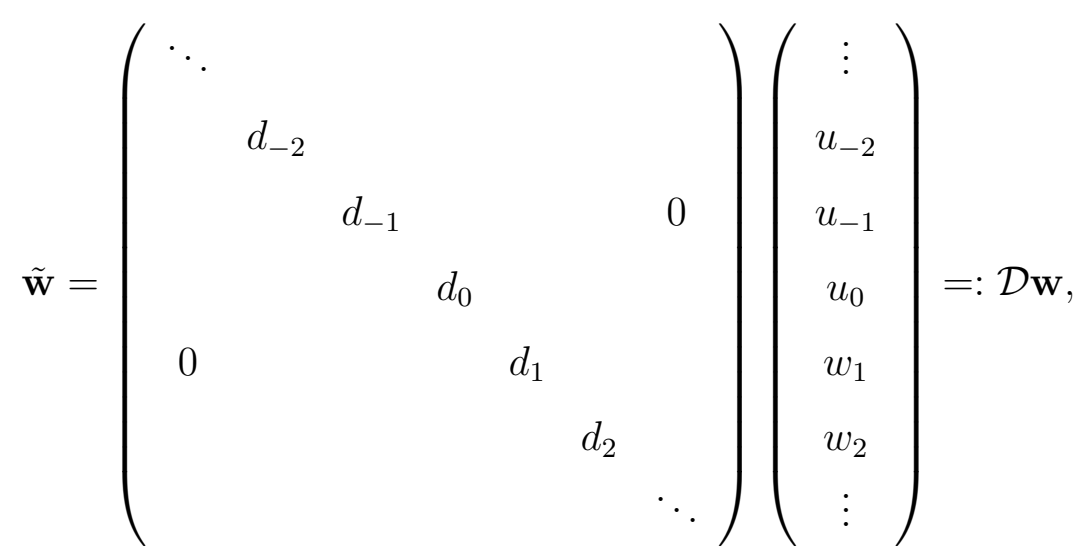

and (33) becomes

$$
\tilde{\mathcal{B}} \tilde{\mathbf{w}}_{t t}=\frac{1}{h^{2}} \tilde{\mathcal{A}} \tilde{\mathbf{w}}, \quad \tilde{\mathcal{B}}=\mathcal{D} \mathcal{B D} \mathcal{D}^{-1}, \quad \tilde{\mathcal{A}}=\mathcal{D} \mathcal{A} \mathcal{D}^{-1}=\tilde{\mathcal{A}}^{*}<0
$$

Without restriction we can assume that $d_{0}=1$. Then $d_{1}$ is determined by the condition that

$$
\left(\begin{array}{ll}
1 & 0 \\
0 & d_{1}
\end{array}\right)\left(\begin{array}{ll}
a_{11} & a_{12} \\
a_{21} & a_{22}
\end{array}\right)\left(\begin{array}{cc}
1 & 0 \\
0 & d_{1}^{-1}
\end{array}\right)=\left(\begin{array}{cc}
a_{11} & d_{1}^{-1} a_{12} \\
d_{1} a_{21} & a_{22}
\end{array}\right)
$$

is symmetric. By $(31),(32)): d_{1}=\sqrt{a_{12} / a_{21}}=c$. The corresponding $2 \times 2$ submatrix of $\mathcal{B}$ becomes

$$
\tilde{\mathcal{B}}_{1}=\left(\begin{array}{ll}
1 & 0 \\
0 & d_{1}
\end{array}\right)\left(\begin{array}{ll}
b_{11} & b_{12} \\
b_{21} & b_{22}
\end{array}\right)\left(\begin{array}{cc}
1 & 0 \\
0 & d_{1}^{-1}
\end{array}\right)=\left(\begin{array}{cc}
b_{11} & b_{12} / c \\
c b_{21} & b_{22}
\end{array}\right)
$$

We want to show that

$$
\frac{1}{2}\left(\tilde{\mathcal{B}}+\tilde{\mathcal{B}}^{*}\right)>0, \quad \tilde{\mathcal{B}}=\mathcal{D B D}^{-1}
$$

We need to prove this only for $\tilde{\mathcal{B}}_{1}$, since the remaining part of $\tilde{\mathcal{B}}$ is identical to $\mathcal{B}$, i.e., diagonal with positive elements. By (29), (30),

$$
\begin{aligned}
& b_{11}=(1-\alpha)\left(\frac{1}{2}+\alpha\right)+\alpha c^{2}>0 \\
& b_{22}=\frac{1}{c^{2}}\left(1-\alpha+\alpha c^{2}\left(\frac{3}{2}-\alpha\right)\right)>0 .
\end{aligned}
$$


The characteristic equation, $\operatorname{det}\left(\frac{1}{2}\left(\tilde{\mathcal{B}}_{1}+\tilde{\mathcal{B}}_{1}^{*}\right)-\mu I\right)=0$, is

$$
\mu^{2}-\mu\left(b_{11}+b_{22}\right)+b_{11} b_{22}-\frac{1}{4}\left(\frac{b_{12}}{c}+c b_{21}\right)^{2}=0
$$

with discriminant

$$
\Delta=\left(b_{11}+b_{22}\right)^{2}-4\left(b_{11} b_{22}-\frac{1}{4}\left(\frac{b_{12}}{c}+c b_{21}\right)^{2}\right)=\left(b_{11}-b_{22}\right)^{2}+\left(\frac{b_{12}}{c}+c b_{21}\right)^{2}>0
$$

The roots are

$$
\mu_{1,2}=\frac{1}{2}\left(b_{11}+b_{22} \pm \sqrt{\Delta}\right) .
$$

Since $b_{11}+b_{22}>0$ and $\Delta>0$, both roots are real and positive if $\left(b_{11}+b_{22}\right)^{2}>\Delta$. We have

$$
\begin{aligned}
\frac{1}{4}\left(\left(b_{11}+b_{22}\right)^{2}-\Delta\right)=b_{11} b_{22}-\frac{1}{4}\left(\frac{b_{12}}{c}+c b_{21}\right)^{2} \\
=\frac{1}{c^{2}}\left((1-\alpha)\left(\frac{1}{2}+\alpha\right)+\alpha c^{2}\right)\left(1-\alpha+\alpha c^{2}\left(\frac{3}{2}-\alpha\right)\right)-\frac{\left(\alpha-\frac{1}{2}\right)^{2}}{4}\left(1-\alpha-\alpha c^{2}\right)^{2} \\
=\frac{1}{c^{2}}\left((1-\alpha)^{2}\left(\frac{1}{2}+\alpha\right)-\frac{\left(\alpha-\frac{1}{2}\right)^{2}}{4}(1-\alpha)^{2}\right)>0 .
\end{aligned}
$$

Thus $\frac{1}{2}\left(\tilde{\mathcal{B}}+\tilde{\mathcal{B}}^{*}\right)$ is positive definite.

If $\alpha=\frac{1}{2}$, then, by (29)-(30), $b_{12}=b_{21}=0$ and $\tilde{\mathcal{B}}=\tilde{\mathcal{B}}^{*}>0$. Since $\tilde{\mathcal{A}}=\tilde{\mathcal{A}}^{*}<0$, there is an energy estimate and the method is stable.

If $\alpha \neq \frac{1}{2}$, then we make an eigenvector expansion. $\tilde{\mathbf{w}}=e^{\lambda t} \tilde{\mathbf{w}}_{0}$ is a solution of (35) if $\lambda, \tilde{\mathbf{w}}_{0}$ are solutions of the eigenvalue problem

$$
\left(\lambda^{2} \tilde{\mathcal{B}}-\frac{1}{h^{2}} \tilde{\mathcal{A}}\right) \tilde{\mathbf{w}}_{0}=0
$$

If we can prove that the eigenvalues $\lambda^{2}$ are real, distinct, and negative, then there are no growing modes and the approximation is stable.

If $\lambda^{2}$ is real, then also $\tilde{\mathbf{w}}_{0}$ is real and the symmetry of $\tilde{\mathcal{A}}$ give us

$$
\lambda^{2}\left\langle\tilde{\mathbf{w}}_{0}, \tilde{\mathcal{B}} \tilde{\mathbf{w}}_{0}\right\rangle=\frac{\lambda^{2}}{2}\left\langle\tilde{\mathbf{w}}_{0},\left(\tilde{\mathcal{B}}+\tilde{\mathcal{B}}^{*}\right) \tilde{\mathbf{w}}_{0}\right\rangle=\frac{1}{h^{2}}\left\langle\tilde{\mathbf{w}}_{0}, \tilde{\mathcal{A}} \tilde{\mathbf{w}}_{0}\right\rangle
$$

where $\langle\mathbf{u}, \mathbf{v}\rangle$ denotes the usual $L_{2}$ inner product. Since $\tilde{\mathcal{A}}<0$ and $\tilde{\mathcal{B}}+\tilde{\mathcal{B}}^{*}>0$, it follows that $\lambda^{2}<0$.

We need 
Lemma 1 Consider a tridiagonal system of equations

$$
\left(\begin{array}{ccccccc}
d_{1} & e_{1} & 0 & \ldots & \ldots & \ldots & 0 \\
l_{2} & d_{2} & e_{2} & 0 & \ldots & \ldots & 0 \\
0 & l_{3} & d_{3} & e_{3} & 0 & \ldots & 0 \\
\ldots & \ldots & \ldots & \ldots & \ldots & \ldots & \ldots \\
0 & \ldots \ldots & \ldots & \ldots & 0 & l_{n} & d_{n}
\end{array}\right)\left(\begin{array}{c}
z_{1} \\
\vdots \\
z_{n}
\end{array}\right)=0
$$

and assume that $\theta=0$ is an eigenvalue. If all $e_{j} \neq 0$ or all $l_{j} \neq 0$, then $\theta$ is a simple eigenvalue (i.e., the invariant subspace has dimension 1).

Proof. Assume that all $e_{j} \neq 0$ and $\theta$ is not a simple eigenvalue. Since the invariant subspace corresponding to $\theta$ has dimension larger than one, we can construct an eigenvector with $z_{1}=0$. But then $z_{2}=z_{3}=\ldots=z_{n}=0$ which is a contradiction. Correspondingly, if all $l_{j} \neq 0$, then there is a solution with $z_{n}=0$ which again implies that $z_{n-1}=z_{n-2}=$ $\ldots=z_{1}=0$. This proves the lemma.

We shall now prove that the eigenvalues $\lambda^{2}$ of (39) are simple for all $0<\alpha<1$. $\lambda^{2}$ is an eigenvalue of (39) if zero is an eigenvalue of the tri-diagonal matrix $Q=h^{2} \lambda^{2} \tilde{\mathcal{B}}-\tilde{\mathcal{A}}$. The conditions of Lemma 1 are violated if there is some value of $\alpha$ where at least one element on the sub-diagonal of $Q$ is zero and one element on the super-diagonal of $Q$ is zero. The only off-diagonal elements of $\tilde{\mathcal{B}}$ are $\tilde{b}_{12}$ and $\tilde{b}_{21}, \mathcal{D}>0$, and $a_{10}=a_{23}=(1-\alpha)+\alpha c^{2}>0$ for all $0 \leq \alpha \leq 1$. Hence, Lemma 1 can only be violated if

$$
h^{2} \lambda^{2} \tilde{b}_{12}-\tilde{a}_{12}=0, \quad \text { and } \quad h^{2} \lambda^{2} \tilde{b}_{21}-\tilde{a}_{21}=0 .
$$

By (29), (30), (31) and (32) we can write (41) as

$$
-h^{2} \lambda^{2}\left(\alpha-\frac{1}{2}\right)(1-\alpha)-c^{2}=0, \quad h^{2} \lambda^{2} \alpha\left(\alpha-\frac{1}{2}\right)-1=0,
$$

i.e.,

$$
\frac{\alpha-1}{\alpha}=c^{2}
$$

which is a contradiction because $c^{2}>0$, but the left hand side is negative for $0<\alpha<1$. Thus the eigenvalues $\lambda^{2}$ of (39) are simple. They are also real because they are solutions of the characteristic equation and smooth functions of $\alpha$. For $\alpha=\frac{1}{2}$ they are real. Therefore they are real for all values of $\alpha$ because they can only become complex at some value $\alpha=\alpha_{0}$ if there is an eigenvalue which is not simple.

This completes the proof of

Theorem 1 The eigenvalues $\lambda^{2}$ of (39) are real, distinct, and negative. Therefore the semi-discrete problem (22), (23) subject to the boundary conditions (24) and the jump conditions (25), (26) is stable. 


\section{The modified equation}

Instead of the complete proof of the last section (which is quite complicated), we shall now use modified equations and show stability for the low and intermediate frequencies. This analysis does not provide any information about the highest frequencies. As we shall see in Section 6, numerical calculations show that our " $A^{T} A$-dissipation" takes care of any instabilities caused by these frequencies. We discuss the technique here because in the multi-dimensional case, the use of modified equations is the only way to obtain stability information. Such an analysis can be found in [2]. Since in [2] we neglected the truncation error in the normal direction, it is important that the restriction of the approximation to $1 \mathrm{D}$ is completely stable.

We introduce the dependent variable

$$
v(x, t)=u(-x, t)
$$

assume $x_{\min } \rightarrow \infty, x_{\max } \rightarrow \infty$, and write (17), (18) as a half-plane problem for the system

$$
\left(\begin{array}{c}
v \\
w
\end{array}\right)_{t t}=\left(\begin{array}{cc}
1 & 0 \\
0 & c^{2}
\end{array}\right)\left(\begin{array}{c}
v \\
w
\end{array}\right)_{x x}, \quad 0 \leq x<\infty, \quad t \geq 0
$$

with the modified jump conditions

$$
\begin{aligned}
v(0, t)+h^{2} \beta v_{x x}(0, t) & =w(0, t)+h^{2} \beta w_{x x}(0, t), \\
v_{x}(0, t)+h^{2} \gamma v_{x x x}(0, t) & =-c^{2}\left(w_{x}(0, t)+h^{2} \gamma w_{x x x}(0, t)\right) .
\end{aligned}
$$

We obtain (43) and (44) from (25) and (26) by adding the leading truncation error term. A simple but tedious calculation shows that

$$
\beta=\frac{(1-\alpha) \alpha}{2}, \quad \gamma=\frac{-2+6 \alpha-3 \alpha^{2}}{6}
$$

We use mode analysis to discuss stability. The general solutions of type

$$
\left(\begin{array}{c}
v(x, t) \\
w(x, t)
\end{array}\right)=e^{s t}\left(\begin{array}{c}
\tilde{v}(x, s) \\
\tilde{w}(x, s)
\end{array}\right), \quad \operatorname{Re}(s) \geq 0,
$$

where $(\tilde{v}(x, s), \tilde{w}(x, s))$ are bounded, are given by

$$
v(x, t)=e^{s t} e^{-s x} v_{0}, \quad w(x, t)=e^{s t} e^{-(s / c) x} w_{0} .
$$

Introducing (45) into the boundary conditions (43), (44), gives us

$$
\begin{aligned}
\left(1+h^{2} \beta s^{2}\right) v_{0}-\left(1+h^{2} \beta s^{2}\right) w_{0} & =0, \\
\left(1+h^{2} \gamma s^{2}\right) v_{0}+c^{2}\left(1+h^{2} \gamma s^{2}\right) w_{0} & =0 .
\end{aligned}
$$


Thus, non-trivial solutions exist if and only if

$$
-\left(1+h^{2} \beta s^{2}\right)\left(1+h^{2} \gamma s^{2}\right)=0 .
$$

Hence, instabilities can only be present for $s=\mathcal{O}(1 / h)$, i.e., for high frequencies. (However, the proof in the previous section shows that this cannot happen).

\section{Solvability of the 2-D discrete jump conditions}

Let the $2 \times 2$ matrix on the left hand side of (16) be $P$. The linear system has a unique solution if $\operatorname{det} P \neq 0$. We have

$$
\operatorname{det} P=g_{0}^{D}\left(\xi_{\Gamma}\right) g_{0}^{N}\left(\xi_{I}-\xi_{\Gamma}\right) / \rho_{I I}+g_{0}^{D}\left(\xi_{I}-\xi_{\Gamma}\right) g_{0}^{N}\left(\xi_{\Gamma}\right) / \rho_{I} .
$$

The coefficients in the Dirichlet formula (8) are

$$
g_{0}^{D}(\xi)=\frac{\left(\xi_{I}-\xi\right)\left(2 \xi_{I}-\xi\right)}{2 \xi_{I}^{2}}+\delta, \quad g_{I}^{D}(\xi)=\frac{\xi\left(2 \xi_{I}-\xi\right)}{\xi_{I}^{2}}-2 \delta, \quad g_{I I}^{D}(\xi)=\frac{\xi\left(\xi-\xi_{I}\right)}{2 \xi_{I}^{2}}+\delta,
$$

where $\delta \approx 0.25$ is a constant that removes the small-cell time step restriction while preserving the second order accuracy, see [3]. The coefficients in the Neumann formula (9) are, (see [2])

$$
g_{0}^{N}(\xi)=\frac{3 \xi_{I}-2 \xi}{2 \xi_{I}^{2}}, \quad g_{I}^{N}(\xi)=\frac{2 \xi_{I}-2 \xi}{\xi_{I}^{2}}, \quad g_{I I}^{N}(\xi)=\frac{\xi_{I}-2 \xi}{2 \xi_{I}^{2}} .
$$

Since $0 \leq \xi_{\Gamma} \leq \xi_{I}$ and $\delta>0$,

$$
0<\delta \leq g_{0}^{D} \leq 1+\delta, \quad 0<\frac{1}{2 \xi_{I}} \leq g_{0}^{N} \leq \frac{3}{2 \xi_{I}} .
$$

Hence

$$
\frac{\delta}{2 \xi_{I}}\left(\frac{1}{\rho_{I}}+\frac{1}{\rho_{I I}}\right) \leq \operatorname{det} P \leq \frac{3(1+\delta)}{2 \xi_{I}}\left(\frac{1}{\rho_{I}}+\frac{1}{\rho_{I I}}\right) .
$$

For all possible directions of the normal, $h \leq \xi_{I} \leq \sqrt{2} h$, and we conclude that

$$
\operatorname{det} P \geq C>0, \quad C=\frac{\delta}{2 \sqrt{2} h}\left(\frac{1}{\rho_{I}}+\frac{1}{\rho_{I I}}\right) .
$$

The fact that the lower bound of $\operatorname{det} P$ is proportional to $\delta$ shows that the $\delta$-term is essential for the solvability of the discrete jump conditions for general locations of the interface relative to the grid. The $\delta$-term was originally designed for Dirichlet boundary conditions to remove the small-cell time-step restriction, cf. [3], and it also serves that purpose here. 


\section{Time integration and $A^{T} A$ dissipation}

The discretized jump conditions (16) can in principle be used to eliminate all ghost points from the discrete approximation of the Laplacians of $u$ and $w(7)$. Since the jump conditions couple the solutions on both sides of the interface, the matrix form of the discrete Laplacian becomes:

$$
\begin{aligned}
\Delta_{h} u\left(\boldsymbol{X}_{I}, t_{n}\right) & =A_{11} \boldsymbol{u}^{n}+A_{12} \boldsymbol{w}^{n}, \\
\Delta_{h} w\left(\boldsymbol{X}_{I I}, t_{n}\right) & =A_{21} \boldsymbol{u}^{n}+A_{22} \boldsymbol{w}^{n},
\end{aligned} \quad A=\left(\begin{array}{cc}
A_{11} & A_{12} \\
A_{21} & A_{22}
\end{array}\right) .
$$

Here, $\boldsymbol{X}_{I}$ and $\boldsymbol{X}_{I I}$ are vectors of all grid point coordinates inside $\Omega_{I}$ and $\Omega_{I I}$, respectively, and $\boldsymbol{u}$ and $\boldsymbol{w}$ are the discrete solutions at those grid points. If all eigenvalues of $A$ are distinct, real, and negative, one can show that the scheme

$$
\left(\begin{array}{c}
\rho_{I}\left(\boldsymbol{u}^{n+1}-2 \boldsymbol{u}^{n}+\boldsymbol{u}^{n-1}\right) / \delta_{t}^{2} \\
\rho_{I I}\left(\boldsymbol{w}^{n+1}-2 \boldsymbol{w}^{n}+\boldsymbol{w}^{n-1}\right) / \delta_{t}^{2}
\end{array}\right)=A\left(\begin{array}{c}
\boldsymbol{u}^{n} \\
\boldsymbol{w}^{n}
\end{array}\right)+\boldsymbol{F}\left(t_{n}\right)
$$

is stable for sufficiently small time steps $\delta_{t}[1]$. However, the embedded boundary approximation of the jump conditions break the symmetry of the matrix $A$ so it is not possible to guarentee this property. In fact, numerical examples (see Section 6) indicate that the above scheme suffers from a mild instability.

In the previous embedded boundary methods for the wave equation subject to Neumann [2] and Dirichlet [3] boundary conditions, we damped the instability by adding a small fourth order term of the type $h^{3} A^{T} A\left(\boldsymbol{u}_{t}, \boldsymbol{w}_{t}\right)^{T}$ to the right hand side of (46). While this technique turned out to work very well in practice for the Neumann and Dirichlet problems, it is not directly amendable to the current problem because the matrix $A$ couples the solutions across the interface. Consequentially, it becomes complicated to evaluate $A^{T}$ on a solution vector without explicitly forming the matrix $A$.

It is not difficult to modify the previous dissipation technique to work with the jump conditions. We first describe the idea for the continuous problem. For the wave equation (1-2) with jump conditions (5-6), we can evaluate the normal derivative of the solutions on either side of the interface as functions of time,

$$
\begin{array}{ll}
\frac{\partial u}{\partial n}(\boldsymbol{x}, t)=: f_{I}(\boldsymbol{x}, t), \quad & \boldsymbol{x} \in \Gamma, t \geq 0, \\
\frac{\partial w}{\partial n}(\boldsymbol{x}, t)=: f_{I I}(\boldsymbol{x}, t), \quad \boldsymbol{x} \in \Gamma, t \geq 0 .
\end{array}
$$

Hence, once the continuous problem with jump conditions has been solved, we can in 
principle re-compute the same solution by solving two uncoupled Neumann problems:

$$
\begin{aligned}
& \rho_{I} u_{t t}=\Delta u+F(\boldsymbol{x}, t), \quad \boldsymbol{x} \in \Omega_{I}, \quad t \geq 0, \\
& \frac{\partial u}{\partial n}(\boldsymbol{x}, t)=f_{I}(\boldsymbol{x}, t), \quad \boldsymbol{x} \in \Gamma, \quad t \geq 0, \\
& u(\boldsymbol{x}, 0)=U_{0}(\boldsymbol{x}), \quad u_{t}(\boldsymbol{x}, 0)=U_{1}(\boldsymbol{x}), \quad \boldsymbol{x} \in \Omega_{I},
\end{aligned}
$$

and

$$
\begin{aligned}
\rho_{I I} w_{t t} & =\Delta w+F(\boldsymbol{x}, t), & \boldsymbol{x} \in \Omega_{I I}, & t \geq 0, \\
\frac{\partial w}{\partial n}(\boldsymbol{x}, t) & =f_{I I}(\boldsymbol{x}, t), & & \boldsymbol{x} \in \Gamma, \quad t \geq 0, \\
w(\boldsymbol{x}, 0)=W_{0}(\boldsymbol{x}), & w_{t}(\boldsymbol{x}, 0) & =W_{1}(\boldsymbol{x}), & \boldsymbol{x} \in \Omega_{I I} .
\end{aligned}
$$

Our basic idea is to use the stabilized embedded boundary scheme for the Neumann problem described in [2], where the forcing functions $f_{I}$ and $f_{I I}$ are computed on the fly during the time evolution.

For conciseness, we only describe the details for the Neumann problem (49), which is discretized on the same Cartesian grid as above, leading to the same set of ghost points $G_{I}$ where the discrete boundary conditions are applied,

$$
B_{i, j}^{N} u^{n}=f_{I}\left(\boldsymbol{x}_{i, j}^{\Gamma}, t_{n}\right), \quad(i, j) \in G_{I} .
$$

The formula (7) for discretizing the Laplacian of $u$ is the same as before, but since the Neumann problem for $u$ is decoupled from $w$, we now get the matrix representation

$$
\Delta_{h} u\left(\boldsymbol{X}_{I}, t_{n}\right)=A_{I} \boldsymbol{u}^{n}+\boldsymbol{b}_{I}^{n},
$$

after all ghost points have been eliminated from (7). The vector $\boldsymbol{b}_{I}$ contains the discrete boundary forcing function corresponding to the forcing function $f_{I}\left(\boldsymbol{x}^{\Gamma}, t\right)$ and is only non-zero at grid points just inside the boundary, see [2] for details.

The stabilized scheme for the Neumann problem is

$$
\begin{aligned}
& \rho_{I}\left(\boldsymbol{u}^{n+1}-2 \boldsymbol{u}^{n}+\boldsymbol{u}^{n-1}\right) / \delta_{t}^{2}= \\
& A_{I} \boldsymbol{u}^{n}+\boldsymbol{b}^{n}+\boldsymbol{F}\left(t_{n}\right)-\epsilon \frac{h^{3}}{\delta_{t}} A_{I}^{T}\left(A_{I} \boldsymbol{u}^{n}+\boldsymbol{b}^{n}-A_{I} \boldsymbol{u}^{n-1}-\boldsymbol{b}^{n-1}\right),
\end{aligned}
$$

where $\epsilon>0$ is a small constant. Numerical experiments indicate that $\epsilon=\mathcal{O}\left(10^{-3}\right)$ is sufficient to allow for very long time integrations $\left(10^{6}\right.$ time steps, or more). The scheme (53) can be recast into an equivalent form that makes it easier to generalize to handle the jump conditions. Let us define an extended solution vector $\overline{\boldsymbol{u}}$, that includes the solution at all interior points as well as the ghost points. Given $\overline{\boldsymbol{u}}$, all values are defined to evaluate the discrete Laplacian $\Delta_{h} u_{i, j}(7)$, at all points $\boldsymbol{x}_{i, j} \in \Omega_{I}$. We write the discrete Laplacian at all these points as $\Delta_{h} \overline{\boldsymbol{u}}$ and arrive at the equivalent method: 
1. Given $\boldsymbol{u}^{n}$, define $\overline{\boldsymbol{u}}^{n}$ by assigning all ghostpoints to satisfy the discretized Neumann boundary condition (51).

2. Update all interior points $\boldsymbol{x}_{i, j} \in \Omega_{I}$ by

$$
\rho_{I}\left(\boldsymbol{u}^{n+1}-2 \boldsymbol{u}^{n}+\boldsymbol{u}^{n-1}\right) / \delta_{t}^{2}=\Delta_{h} \overline{\boldsymbol{u}}^{n}+\boldsymbol{F}\left(t_{n}\right)-\epsilon \frac{h^{3}}{\delta_{t}} A_{I}^{T}\left(\Delta_{h} \overline{\boldsymbol{u}}^{n}-\Delta_{h} \overline{\boldsymbol{u}}^{n-1}\right) .
$$

Using this formulation shows that the matrix $A_{I}$ is only needed to evaluate the dissipation term, and only for the matrix-vector product $A_{I}^{T} \boldsymbol{y}$, where $\boldsymbol{y}=\Delta_{h} \overline{\boldsymbol{u}}^{n}-\Delta_{h} \overline{\boldsymbol{u}}^{n-1}$. Note that the boundary forcing $f_{I}\left(\boldsymbol{x}^{\Gamma}, t\right)$ only influences the ghost point values and has no bearing on $A_{I}^{T}$.

To satisfy the discrete jump conditions (14)-(15), it appears that we must first calculate a corresponding value of $f_{I}$ to use in (51). Given $\boldsymbol{u}^{n}$, this can be achieved by first calculating the values at all ghost points $G_{I}$ by solving the $2 \times 2$ linear system (16). We can then use the embedded boundary formula (51) to evaluate $f_{I}$. However, it is a trivial exercise to see that enforcing (51) with this $f_{I}$ results in the same ghost point values as we started with. Hence, it is not necessary to calculate $f_{I}$, and we can simply replace the inhomogeneous Neumann condition by the discrete jump conditions (16). The same principle applies to the Neumann problem in $\Omega_{I I}$ and we arrive at the damped scheme for the problem with jump conditions:

1. Given $\boldsymbol{u}^{n}$ and $\boldsymbol{w}^{n}$, define $\overline{\boldsymbol{u}}^{n}$ by assigning all ghostpoints $u_{i, j}$ to satisfy the discrete jump conditions along normals going through $(i, j) \in G_{I}$,

$$
\begin{aligned}
B_{i, j}^{D} u^{n} & =V_{i, j}^{D} w^{n}, \\
\frac{1}{\rho_{I}} B_{i, j}^{N} u^{n} & =-\frac{1}{\rho_{I I}} V_{i, j}^{N} w^{n},
\end{aligned}
$$

2. Given $\boldsymbol{u}^{n}$ and $\boldsymbol{w}^{n}$, define $\overline{\boldsymbol{w}}^{n}$ by assigning all ghostpoints $w_{i, j}$ to satisfy the discrete jump conditions along normals going through $(i, j) \in G_{I I}$,

$$
\begin{aligned}
B_{i, j}^{D} w^{n} & =V_{i, j}^{D} u^{n}, \\
\frac{1}{\rho_{I I}} B_{i, j}^{N} w^{n} & =-\frac{1}{\rho_{I}} V_{i, j}^{N} u^{n},
\end{aligned}
$$

3. Update all interior points $\boldsymbol{x}_{i, j} \in \Omega_{I}$ by

$$
\rho_{I}\left(\boldsymbol{u}^{n+1}-2 \boldsymbol{u}^{n}+\boldsymbol{u}^{n-1}\right) / \delta_{t}^{2}=\Delta_{h} \overline{\boldsymbol{u}}^{n}+\boldsymbol{F}\left(t_{n}\right)-\epsilon \frac{h^{3}}{\delta_{t}} A_{I}^{T}\left(\Delta_{h} \overline{\boldsymbol{u}}^{n}-\Delta_{h} \overline{\boldsymbol{u}}^{n-1}\right) .
$$

4. Update all interior points $\boldsymbol{x}_{i, j} \in \Omega_{I I}$ by

$$
\rho_{I I}\left(\boldsymbol{w}^{n+1}-2 \boldsymbol{w}^{n}+\boldsymbol{w}^{n-1}\right) / \delta_{t}^{2}=\Delta_{h} \overline{\boldsymbol{w}}^{n}+\boldsymbol{F}\left(t_{n}\right)-\epsilon \frac{h^{3}}{\delta_{t}} A_{I I}^{T}\left(\Delta_{h} \overline{\boldsymbol{w}}^{n}-\Delta_{h} \overline{\boldsymbol{w}}^{n-1}\right) .
$$


Here, $A_{I I}$ denotes the matrix representation of the discrete Laplacian subject to discrete Neumann conditions in $\Omega_{I I}$, and $\overline{\boldsymbol{w}}^{n}$ is the extended solution vector holding the solution at all interior grid points of $\Omega_{I I}$ as well as at the ghost points $G_{I I}$. Note that the matrices $A_{I}$ and $A_{I I}$ do not need to be formed explicitly, cf. [2].

The wave equation subject to jump condition is equivalent to the two uncoupled wave equations subject to Neumann boundary conditions (49)-(50), and the theory in [2] shows that the $h^{3} A^{T} A u_{t}$ dissipation term inflicts an error which is $\mathcal{O}\left(h^{2}\right)$ for these problems. Hence, we conclude that the dissipation term also inflicts an $\mathcal{O}\left(h^{2}\right)$ error for the wave equation with jump conditions.

\section{$6 \quad$ Numerical examples}

To test the accuracy of the numerical scheme, we begin by considering electromagnetic scattering of a plane incident wave by a dielectric circular cylinder of radius $R$. In this section, we follow the notation of electromagnetics and let $\kappa_{e}$ denote the relative permittivity of the dielectric material and assume unit relative permissivity. By assuming $\mathrm{TE}_{z}$ polarization and scaling time to obtain unit speed of light in vacuum, we arrive at the following problem for the $z$-component of the magnetic field,

$$
\begin{array}{rlrl}
\frac{\partial^{2} H^{(z)}}{\partial t^{2}} & =\Delta H^{(z)}, & R^{2}<x^{2}+y^{2}<\infty, \\
\frac{\partial^{2} H^{(z)}}{\partial t^{2}}=\frac{1}{\kappa_{e}} \Delta H^{(z)}, & x^{2}+y^{2}<R^{2},
\end{array}
$$

subject to the jump conditions

$$
\left[H^{(z)}\right]=0, \quad\left[\frac{1}{\kappa_{e}} \frac{\partial H^{(z)}}{\partial n}\right]=0, \quad x^{2}+y^{2}=R^{2} .
$$

Here, $\partial / \partial n$ denotes the normal derivative on the boundary of the dielectric cylinder and $\kappa_{e}=1$ outside the cylinder. There is an analytical solution of this problem due to Mie (see, for example, [13] p. 667). Let the incident wave have angular frequency $\omega$ and wave number $k$,

$$
H_{I}^{(z)}(x, y, t)=e^{i(k x-\omega t)}
$$




\begin{tabular}{c|c|c|c|c|c}
$N$ & $\mathrm{~h}$ & $u_{\text {err }}$ & $u_{\text {err }}, A^{T} A$ & $w_{\text {err }}$ & $w_{\text {err }}, A^{T} A$ \\
\hline 201 & $1.5 \times 10^{-2}$ & $4.50 \times 10^{-2}$ & $4.47 \times 10^{-2}$ & $2.07 \times 10^{-2}$ & $2.09 \times 10^{-2}$ \\
401 & $7.5 \times 10^{-3}$ & $1.12 \times 10^{-3}$ & $1.11 \times 10^{-2}$ & $5.44 \times 10^{-3}$ & $5.49 \times 10^{-3}$ \\
801 & $3.75 \times 10^{-3}$ & $2.81 \times 10^{-3}$ & $2.80 \times 10^{-3}$ & $1.30 \times 10^{-3}$ & $1.31 \times 10^{-3}$ \\
\hline
\end{tabular}

Table 1: Max error at time $T=10.0$ for the case $\kappa_{e}=2$ inside the dielectric cylinder $\left(u_{e r r}\right)$ and outside of it $\left(w_{e r r}\right)$, with $A^{T} A$ dissipation $\left(\epsilon=10^{-3}\right)$ and without dissipation.

In polar coordinates $(\rho, \theta)$, the incident, scattered and transmitted fields are

$$
\begin{array}{ll}
H_{I}^{(z)}=e^{i \omega t} \sum_{n=-\infty}^{\infty} i^{-n} J_{n}(k \rho) e^{i n \theta}, & \rho>R \\
H_{S}^{(z)}=e^{i \omega t} \sum_{n=-\infty}^{\infty} i^{-n} a_{n} H_{n}^{(2)}(k \rho) e^{i n \theta}, & \rho>R, \\
H_{D}^{(z)}=e^{i \omega t} \sum_{n=-\infty}^{\infty} i^{-n} b_{n} J_{n}(m k \rho) e^{i n \theta}, & \rho<R,
\end{array}
$$

where $m=\sqrt{\kappa_{e}}, J_{n}$ is the Bessel function of the first kind of order $n$, and $H^{(2)}$ is the Hankel function of the second kind of order $n$ (corresponding to waves propagating outwards). The coefficients $a_{n}$ and $b_{n}$ are given in [13] p. 667 .

The scattering problem was solved numerically for the case $R=1, \kappa_{e}=2, \omega=k=2 \pi$. The computational domain was the square $-1.5 \leq x \leq 1.5,-1.5 \leq y \leq 1.5$, and the exact solution was imposed as a Dirichlet boundary condition on the outer boundary. The exact solution was also imposed as initial condition. The error in the numerical solution measured in max norm over all internal grid points was evaluated at times $T=10.0$ and $T=200.0$, see Tables 1 and 2, respectively. Note that the error is second order accurate at both times and of the same order of magnitude for the same grid sizes. Studying the error as function of time, Figure 4, reveals that the $A^{T} A$ dissipation is only necessary for long-time computations. Furthermore, there is no noticable growth of the error after long times when the dissipation is used, indicating that the damping is very weak.

As a second test, we consider the same geometry as above, but reduce the wave speed inside the cylinder by setting $\kappa_{e}=10$. Hence the wave length inside the cylinder will be a factor $\sqrt{10}$ smaller than outside of it. For this reason, a smaller grid size is needed to resolve the solution and the grid with $N=201$ is no longer adequate. The errors in the solution at times $T=10$ and $T=200$ are given in Tables 3 and 4, respectively. Even though the errors are larger in this case, they are still second order accurate at both times and of the same order of magnitude for the same grid sizes. The solution at time $T=10$ is shown in Figure 5. 


\begin{tabular}{c|c|c|c|c}
$N$ & $\mathrm{~h}$ & time steps & $u_{\text {err }}, A^{T} A$ & $w_{\text {err }}, A^{T} A$ \\
\hline 201 & $1.5 \times 10^{-2}$ & 26,666 & $3.38 \times 10^{-2}$ & $3.57 \times 10^{-2}$ \\
401 & $7.5 \times 10^{-3}$ & 53,333 & $8.42 \times 10^{-3}$ & $7.82 \times 10^{-3}$ \\
801 & $3.75 \times 10^{-3}$ & 106,666 & $2.31 \times 10^{-3}$ & $1.97 \times 10^{-3}$ \\
\hline
\end{tabular}

Table 2: Max error at time $T=200.0$ for the case $\kappa_{e}=2$ inside the dielectric cylinder $\left(u_{e r r}\right)$ and outside of it $\left(w_{e r r}\right)$. The coefficient in the $A^{T} A$ dissipation was $\epsilon=10^{-3}$.

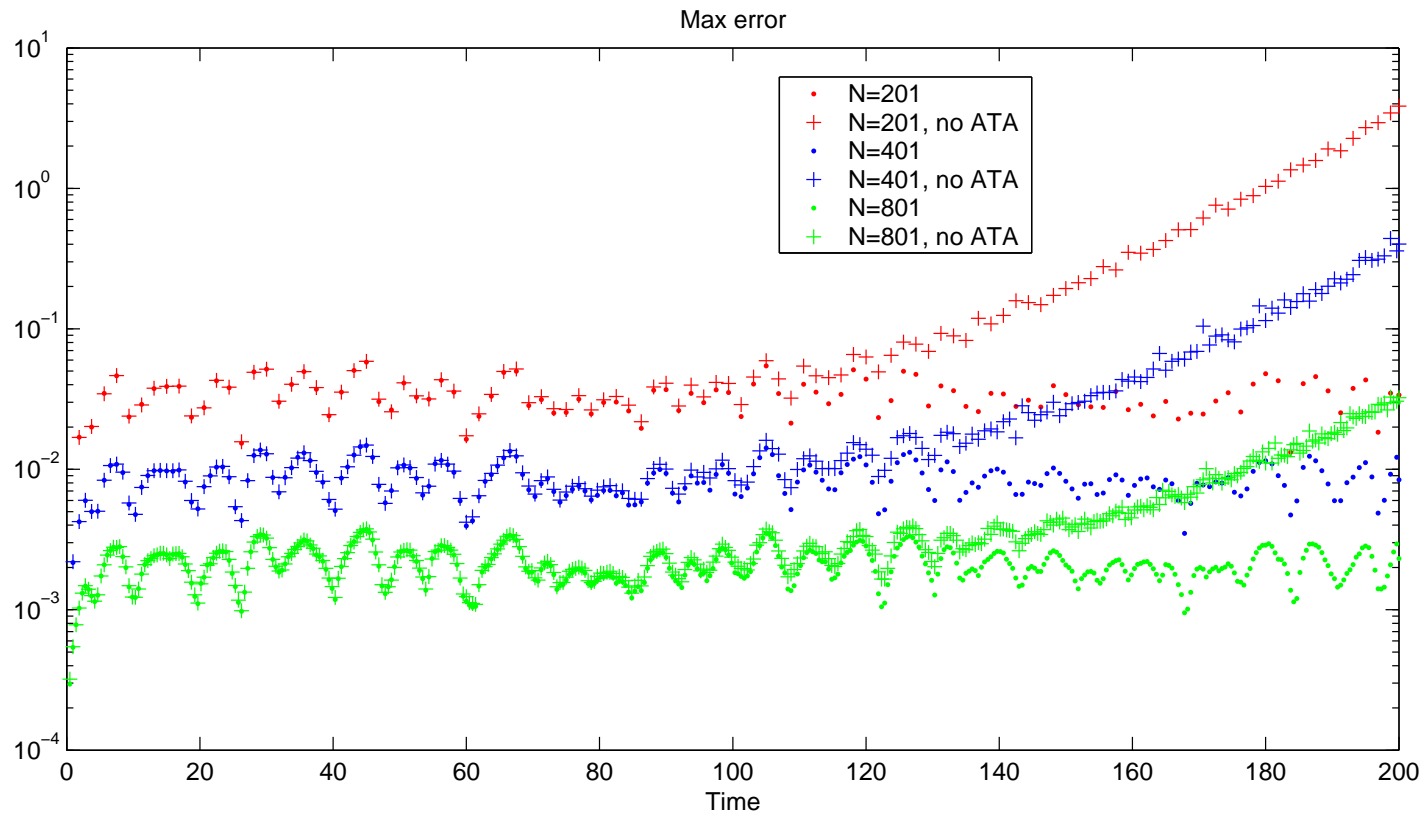

Figure 4: The max error inside the dielectric cylinder $\left(u_{e r r}\right)$ as function of time for the case $\kappa_{e}=2$, for different grid sizes, with and without $A^{T} A$ dissipation.

\begin{tabular}{c|c|c|c|c|c}
$N$ & $\mathrm{~h}$ & $u_{\text {err }}$ & $u_{\text {err }}, A^{T} A$ & $w_{\text {err }}$ & $w_{\text {err }}, A^{T} A$ \\
\hline 401 & $7.5 \times 10^{-3}$ & $2.17 \times 10^{-1}$ & $2.16 \times 10^{-1}$ & $6.52 \times 10^{-2}$ & $6.48 \times 10^{-2}$ \\
801 & $3.75 \times 10^{-3}$ & $5.48 \times 10^{-2}$ & $5.47 \times 10^{-2}$ & $1.62 \times 10^{-2}$ & $1.61 \times 10^{-2}$ \\
\hline
\end{tabular}

Table 3: Max error at time $T=10.0$ for the case $\kappa_{e}=10$ inside the dielectric cylinder $\left(u_{\text {err }}\right)$ and outside of it $\left(w_{\text {err }}\right)$, with $A^{T} A$ dissipation $\left(\epsilon=10^{-3}\right)$ and without dissipation. 


\begin{tabular}{c|c|c|c|c}
$N$ & $\mathrm{~h}$ & time steps & $u_{\text {err }}, A^{T} A$ & $w_{\text {err }}, A^{T} A$ \\
\hline 401 & $7.5 \times 10^{-3}$ & 53,333 & $2.03 \times 10^{-1}$ & $7.16 \times 10^{-2}$ \\
801 & $3.75 \times 10^{-3}$ & 106,666 & $5.81 \times 10^{-2}$ & $1.75 \times 10^{-2}$ \\
\hline
\end{tabular}

Table 4: Max error at time $T=200.0$ for the case $\kappa_{e}=10$ inside the dielectric cylinder $\left(u_{e r r}\right)$ and outside of it $\left(w_{e r r}\right)$. The coefficient in the $A^{T} A$ dissipation was $\epsilon=10^{-3}$.

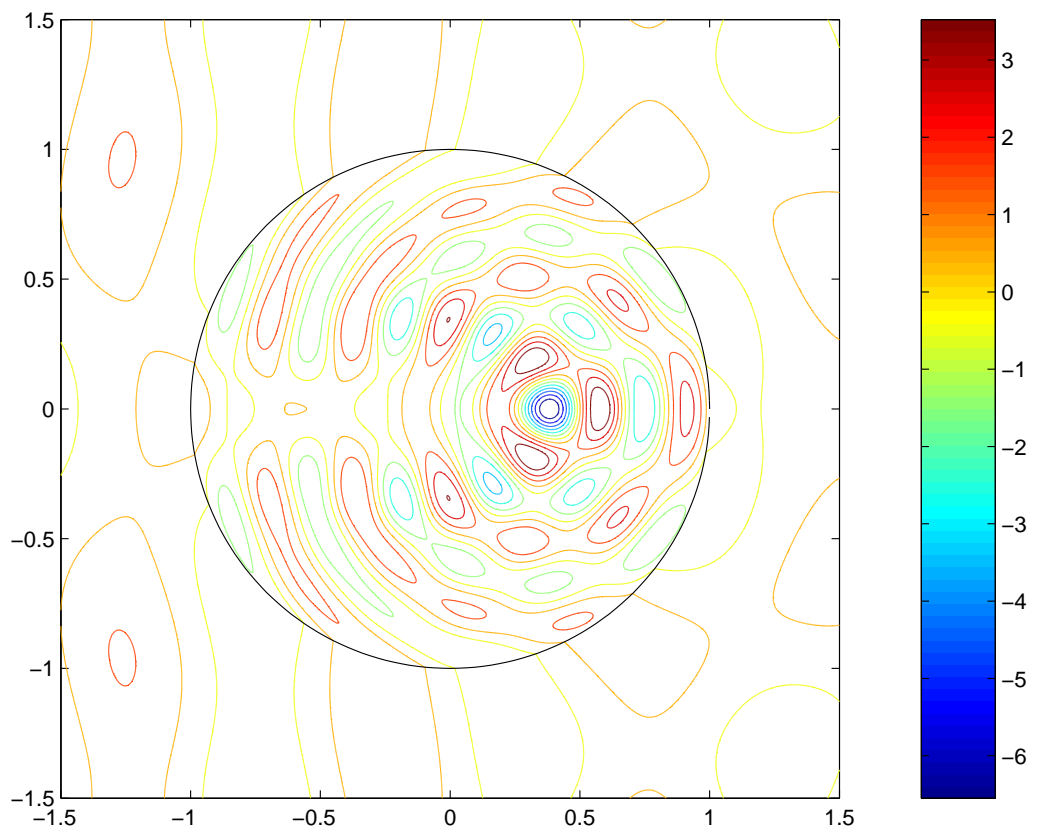

Figure 5: The Mie scattering solution at time $T=10$ with $\kappa_{e}=10$. Note the focusing of the wave inside the cylinder. 


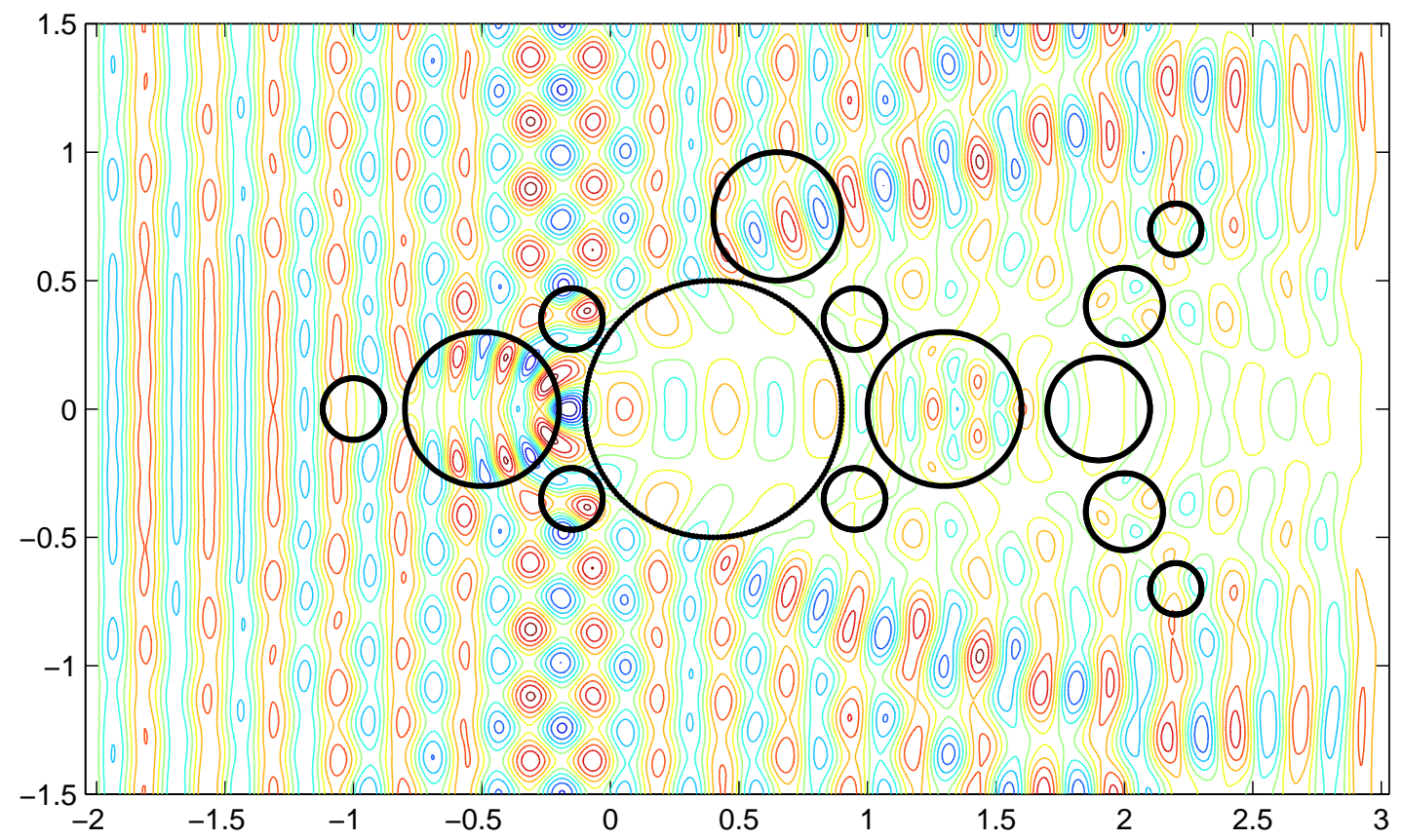

Figure 6: An incoming planar wave scattered by several bubbles with both larger and smaller wave speed compared to the ambient media.

To illustrate that the method generalizes to many subdomains, we consider the scattering of a plane incoming wave on a media with different wave speeds inside each bubble, see Figure 6. This calculation was started from homogeneous initial data, and driven on the left boundary by the data $u(-2, y, t)=\sin (8 \pi t)$. The remaining outer boundaries had homogeneous Neumann conditions. The solution is shown at time $T=5$. The ambient media has unit wave speed, so this time corresponds closely to the first arrival of the solution at the right boundary. Note that the speed inside the top bubble (which breaks the otherwise symmetrical configuration) equals the unit speed in the ambient media, but the jump conditions are enforced across its interface. Never the less, the contour lines of the numerical solution display a high degree of symmetry about the $y=0$ axis, indicating that the truncation errors in the jump conditions are very small.

\section{Conclusions}

We have developed a second order accurate embedded boundary method for the twodimensional wave equation with discontinuous coefficients. The current method uses the same grid size throughout the computational domain, but as the jump in wave speed across the interface gets larger, it becomes obvious that this approach either over-resolves the solution on one side of the interface, or under-resolves it on the other side. It would 
therefore be desirable to extend the method to handle different mesh sizes on different sides of the interface; some initial steps have been taken in this direction. We also expect to generalize the method to handle the more complicated jump conditions associated with the elastic wave equation with discontinuous coefficients.

\section{References}

[1] H.-O. Kreiss, N. A. Petersson, and J. Yström. Difference approximations for the second order wave equation. SIAM J. Numer. Anal., 40:1940-1967, 2002.

[2] H.-O. Kreiss, N. A. Petersson, and J. Yström. Difference approximations of the Neumann problem for the second order wave equation. SIAM J. Numer. Anal., 42:1292-1323, 2004.

[3] H.-O. Kreiss and N. A. Petersson. A second order accurate embedded boundary method for the wave equation with Dirichlet data. UCRL-JRNL 202686, Lawrence Livermore National Lab, 2004. (to appear in SIAM J. Sci. Comput.).

[4] R. B. Pember, J. B. Bell, P. Collella, W. Y. Crutchfield, and M. Welcome. An adaptive Cartesian grid method for unsteady compressible flow in irregular regions. J. Comput. Phys., 120:278, 1995.

[5] M. J. Berger, C. Helzel, and R. J. LeVeque. H-Box methods for the approximation of hyperbolic conservation laws on irregular grids. SIAM J. Numer. Anal., 41:893-918, 2003.

[6] A. Ditkowski, K. Dridi, and J. S. Hesthaven. Convergent Cartesian grid methods for Maxwell's equations in complex geometries. J. Comput. Phys., 170:39-80, 2001.

[7] B. Gustafsson, H.-O. Kreiss, and J. Oliger. Time dependent problems and difference methods. Wiley-Interscience, 1995.

[8] G. Browning, H-.O. Kreiss, and J. Oliger. Mesh refinement. Math. Comp., 27:29-39, 1973.

[9] D. L. Brown. A note on the numerical solution of the wave equation with piecewise smooth coefficients. Math. Comput., 42(166):369-391, 1984.

[10] A. N. Tikhonov and A. A. Samarskil. Homogeneous difference schemes. Zh. Vychisl. Mat. i Mat. Fiz., 1:5-63, 1961.

[11] C. Zhang and R. LeVeque. The immersed interface method for acoustic wave equations with discontinuous coefficients. Wave Motion, 25:237-263, 1997. 
[12] G. Mie. Beiträge zur optik trüber medien, speziell kolloidaler metallösungen. Annalen der Physik, 25(3):377-445, 1908.

[13] C. A. Balanis. Advanced Engineering Electromagnetics. Wiley, New York, 1989. 